Bull. Mater. Sci., Vol. 3, Number 2, July 1981 pp. 109-117. (c) Printed in India.

\title{
Structural aspects of alpha-omega transformation in group IV transition metals and alloys
}

\author{
Y K VOHRA, S K SIKKA and R CHIDAMBARAM \\ Neutron Physics Division, Bhabha Atomic Research Centre, Trombay, \\ Bombay 400 085, India
}

MS received 9 January 1981

\begin{abstract}
The three most common crystal structures encountered in group IV transition metals $\mathrm{Ti}, \mathrm{Zr}$, and $\mathrm{Hf}$ and alloys based on them, under diff(rent temperature, pressure and alloy concentration conditions, are hcp $(a), \operatorname{bcc}(\beta)$ and simple hexagonal ( $\omega)$. Although the structural relations of $a \approx \beta$ and $\beta \approx \omega$ transformations are well understood, the same is not true for $a \rightarrow \omega$ phase change, which occurs at high pressures. We have done high pressure experiments on Ti-V alloys, followed by electron diffraction to study this. These patterns from pressure treated foils of alloys $T i_{95} V_{5}$ and $\mathrm{Ti}_{91} \mathrm{~V}_{9}$ showed the presence of $\beta$-phase with four $\omega$ variants. Some of them showed the existence of all three phases, $a, \beta$ and $\omega$, with the number of variants given by the lattice correspondence matrix, derived through the orientation relations of $a \rightarrow \beta$ and $\beta \rightarrow \omega$. This is a clear evidence that the $a \rightarrow \omega$ transformation proceeds via the $\beta$-phase. The atomic rearrangements required for $a \rightarrow \omega$ are found to be much smaller if the path is via the $\beta$-phase, rather than the earlier model of Silcock.
\end{abstract}

Keywords. Alpha-omega phase transformation; structure; high pressure; orientation relations; transition metals.

\section{Introduction}

The three most common crystal structures encountered in group IV transition metals $\mathrm{Ti}, \mathrm{Zr}$ and $\mathrm{Hf}$ and alloys based on them with other $d$-electron-rich transition elements (i.e., V, Nb, Mo, Ta, etc.,) are hcp $(\alpha)$, bcc $(\beta)$ and simple hexagonal ( $\omega)$. The $\beta$-phase, which is the high temperature phase in all cases, transforms to either $\alpha$ or $\omega$ in alloys on suitable thermal treatments (Hickman 1969). $\quad \alpha \rightarrow \omega$ transformamation is known to occur under high pressures (Jamieson 1963; Vohra 1978). The structural relations between $\beta$ and $\alpha$ and $\beta$ and $\omega$ phases are rather well understood. The hep case represents a zone centre or long wavelength instability on $\{112\} \beta$ planes in the $\langle 111\rangle_{\beta}$ direction followed by a $[110]_{\beta}:\langle 1 \overline{10}\rangle_{\beta}$ zone boundary shear, which doubles the unit cell, while $\omega$ transformation requires a short wavelength or zone boundary instability $[112]_{\beta}:\langle 1 \overline{10}\rangle_{\beta}$, tripling the unit cell (Mose et al 1973).

However, the st iuctural relations between the $\alpha$ and $\omega$-phases are not equally well understood. For this, there have been two suggestions in literature. The first was 
by Silcock (1958), which involves large atomic movements to generate the $\omega$ lattice from the $\alpha$-lattice. The second is by Usikov and Zilbershtein (1973) that $\alpha \rightarrow \omega$ transformation proceeds as $\alpha \rightarrow \beta \rightarrow \omega$. This conclusion was based on the orientation relations (OR's) between $\alpha$ and $\omega$ phases obtained by selected area electron diffraction (SAD) study done on pressure treated $\mathrm{Ti}$ and $\mathrm{Zr}$ foils. It was shown that the experimentally observed OR's can be explained by a lattice correspondence matrix which can be written as a product of $\alpha \rightarrow \beta$ and $\beta \rightarrow \omega$ matrices. Thereby it was concluded that the $\beta$-phase must have been the intermediate state during $\alpha \rightarrow \omega$ transformation. Obviously, such a representation is not unique as the orientation relations of variant II of $\alpha-\omega$ given by this matrix correspondance method and not observed experimentally by Usikov and Zilbershtein (1973), were the same as predicted by Silcock (1958) for her mechanism of $\omega \rightarrow \alpha$ and observed by Flower and Swann (1973). To decide between the two models, we have done high pressure studies on Ti-V alloys combined with $\mathrm{x}$-ray diffraction and electron microscopy. In situ high pressure diffraction studies were not required as the pressure induced phases are retained in a metastable manner at ambient conditions in these systems.

\section{Experimental}

The alloy compositions used in these experiments were decided with the help of the known phase diagram of Ti-V system (Collings 1975). It is now well-known that by suitable addition of a $\beta$-stabilizing element ( $V$ in this case), it is possible to bring the free energies of the three phases viz., the $\alpha$, the $\beta$ and $\omega$, very close to each other. It was then felt by us that choosing alloys near this composition but with only $\alpha$-phase present, the application of pressure on it would show the sequence of $\alpha \rightarrow \omega$ transformation. The experiments therefore, were first carried out on $T i_{91} V_{9}$ and $T i_{95} V_{5}$ alloys. The alloys were prepared by arc melting the constituent elements (Vohra et al 1980 for more details). The homogenised alloys were fabricated in thin foils, which were subsequently water-quenched from the $\beta$-phase to produce the martensitic $\alpha^{\prime}$-phase*. The $\alpha^{\prime}$-martensite was found to be complete in $\mathrm{Ti}_{95} \mathrm{~V}_{5}$, while in the $\mathrm{Ti}_{01} \mathrm{~V}_{9}$ alloy, electron diffraction showed that small quantities of the $\beta$ and $\omega$ were present in some regions.

The foils of these alloys were subjected to pressure for 24 hr by a Bridgman opposed anvil high pressure apparatus. The $\mathrm{Ti}_{95} \mathrm{~V}_{5}$ alloy was kept at $80 \mathrm{Kbar}$ and $\mathrm{Ti}_{\mathrm{y1}} \mathrm{V}_{9}$ at $63 \mathrm{Kbar}$. These were subsequently thinned electrolytically for electron microscopy.

\section{Results}

$X$-ray diffraction of the pressure treated foils showed the presence of the $\omega$-phase along with $\alpha^{\prime}$-phase, but the presence of $\beta$ phase could not be ascertained as all the $\beta$ reflections are common to the $\omega$-phase.

Transmission electron micrographs showed untransformed regions having initial martensitic morphology as well as transformed regions (figure 1). The SAD patterns obtained from these transformed region were of three types. These either contained spots from all the three phases $\left(\alpha^{\prime}, \beta\right.$ and $\left.\omega\right)$ or from two phases, $(\beta, \omega)$, or

*We follow notation of Williams (1973) and designate martensiticaily formed $a$ as $a^{\prime}$. 

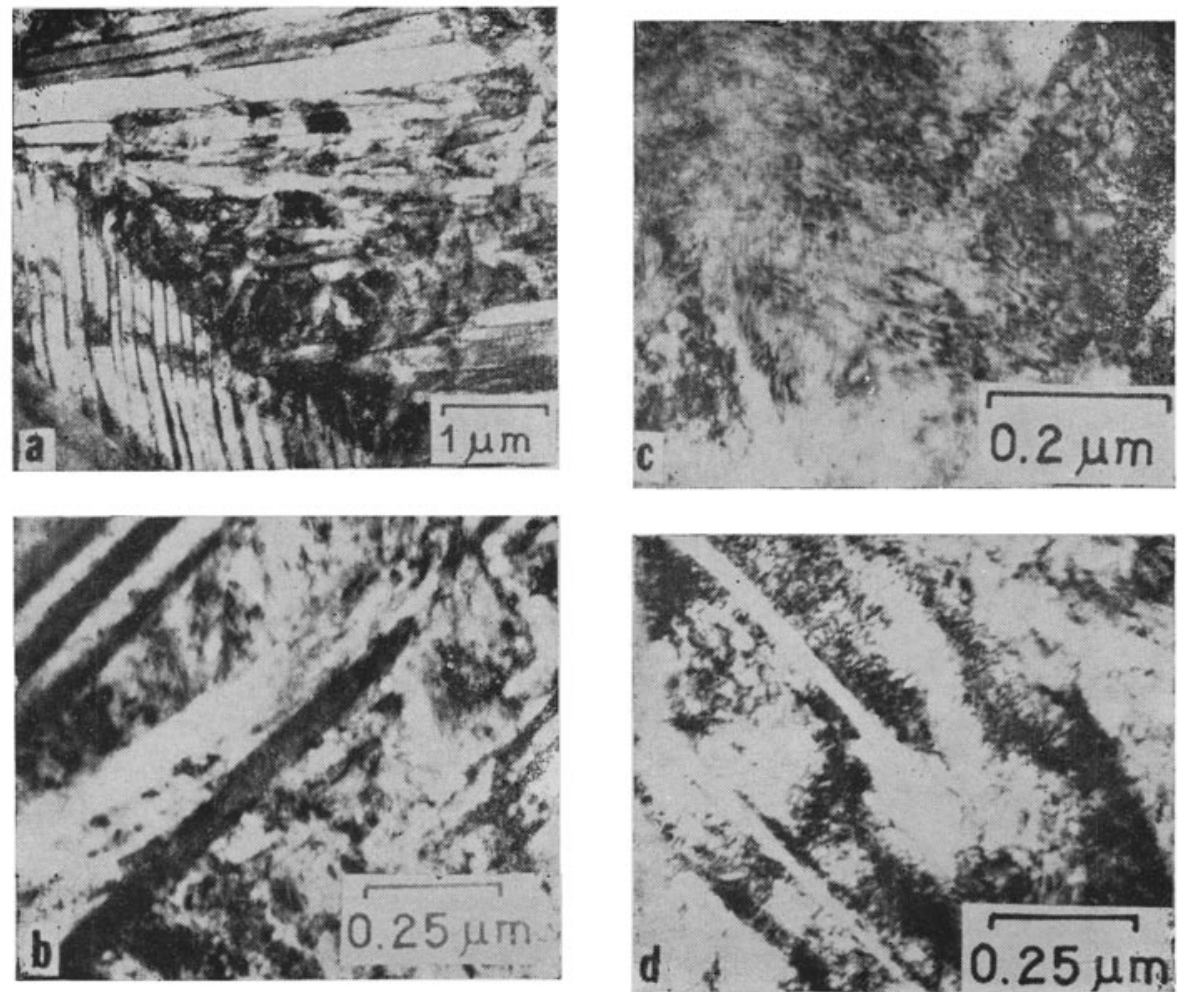

Figure 1. (a) $\mathrm{Ti}_{95} \mathrm{~V}_{5}$ bright field micrograph showing the initial martensitic morphology (b) same for $\mathrm{Ti}_{91} \mathrm{~V}_{9}$. (c) and (d) are taken from the samples (a) and (b) after pressure treatment and show the transformed morphology. 

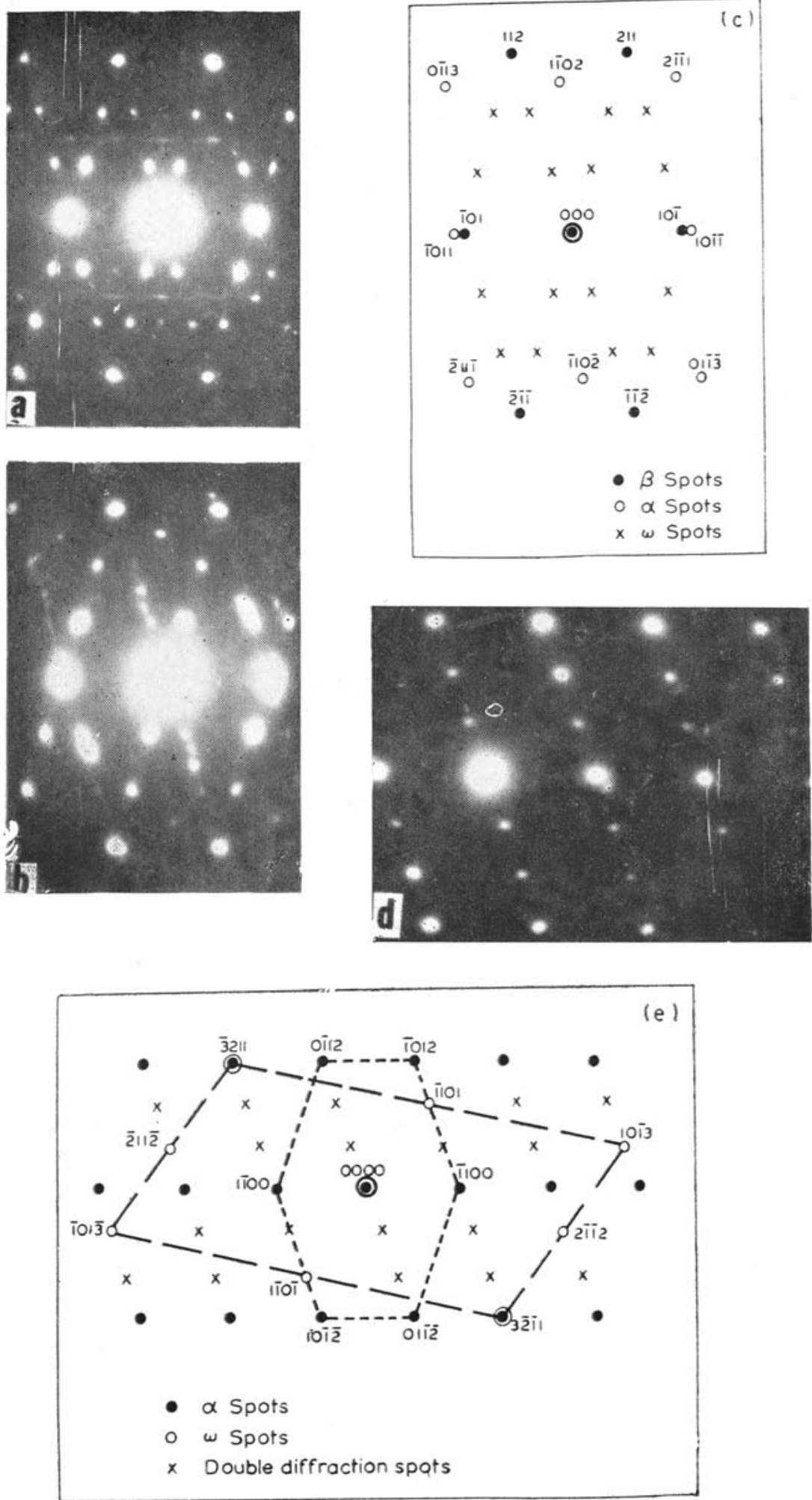

Figure 2. $S A D$ patterns after pressure treatment. (a) $T_{i_{95}} V_{5}$ (zone axis $[\mathbf{1} \overline{3} \mathbf{1}]_{\beta}$ ), (b) $\mathrm{Ti}_{91} \mathrm{~V}_{9}$ (zone axis $\left[\begin{array}{lll}1 & \overline{3} & 1\end{array}\right]_{\beta}$ ), (c) Key to (b). (d) $\mathrm{Ti}_{9,1} \mathrm{~V}_{9}$ for $\left(a^{\prime}+\omega\right)$ region (zone axis $[\overline{2} \overline{4} \overline{3}]_{\beta}$ ), and (e) Key to (d). 

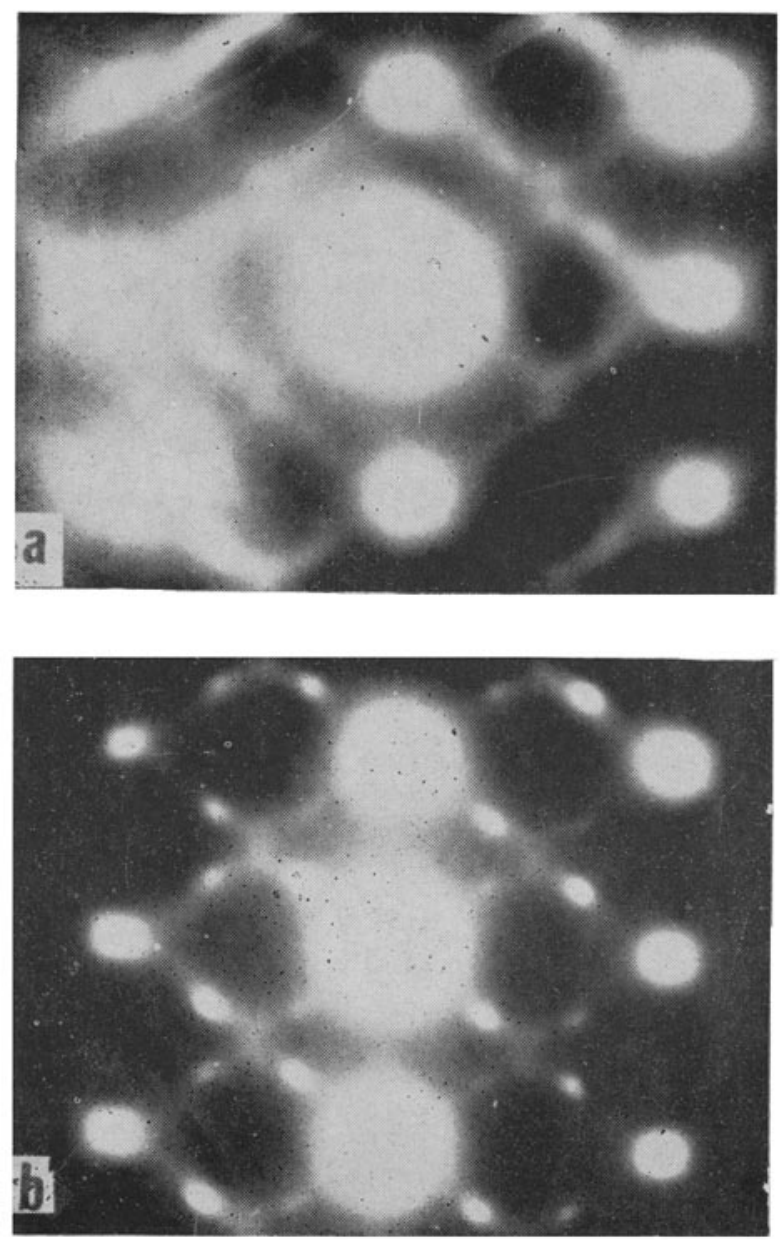

Figure 3. SAD patterns for $T i_{81} \vee_{19}$ alloy (zone axis [110] $\beta$ ) (a) As quenched, (b) after pressure treatment. 
$(\alpha, \omega)$ (figure 2). The amount of $\beta$ was found to decrease with increase in prescure for the $T i_{91} V_{9}$ alloy. This coupled with the fact that $\beta-\omega$ patterns showed the usual four variants, is a direct proof that $\alpha \rightarrow \omega$ transformation proceed via the $\beta$-phase. The four variants arise because the (111) plane collapse in the $\beta$-phase, which leads to $\omega$-phase (De Fontaine 1970), can occur in any one of the four crystallographically equivalent $\{111\}$ planes. Further, both $\alpha-\omega$ variants, predicted by the lattice correspondence matrix of Usikov and Zilbershtein (1973) were seen in the diffraction patterns.

Now the question arises, if the structural sequence $\alpha \rightarrow \beta \rightarrow \omega$ is correct, the $\beta \rightarrow \omega$ transformation should occur under pressure from samples containing $\beta$-phase only. Before this investigation, there was no direct proof of this. Only lowering of superconducting temperature $T_{c}$ was observed in $\mathrm{Zr}-\mathrm{Nb}$ alloys on application of pressure and Afonikova et al (1973) attribute this to the onset of $\omega$-phase. To check this, we prepared $\mathrm{Ti}_{81} \mathrm{~V}_{19}$ and $\mathrm{Ti}_{72} \mathrm{~V}_{28}$ alloys in $\beta$ phase and subjected them to high pressures. The SAD patterns before and after pressure treatment are shown in figure 3. These again clearly show that $\beta \rightarrow \omega$ does progress under pressure.*

\section{Structural mechanism of $\alpha-\omega$ transformation}

From SAD patterns which could be indexed as a superposition of the three phases $v i z$., the $\alpha$, the $\beta$, and the $\omega$, the unique orientation relations between the three phases were identified as,

$\left.\begin{array}{ccccc}(0001)_{a} & || & (110)_{\beta} & || & (0111)_{\omega} \\ {[10 \overline{1} 0]_{a}} & || & {[\overline{1} 11]_{\beta}} & \mid & {[\overline{1011}]_{\omega}}\end{array}\right\}$ variant I

The structural relationship for the variant II is shown in figure 4. The atomic arrangements in mutually parallel planes, $(0001)_{a},(011)_{\beta}$ and $(11 \overline{20})_{\omega}$ are quite similar and require small atomic adjustments to go from one to the other. The transformation mechanism, which is now consistent with the martensitic nature of its kinetics (Vohra 1978), can be described by the Burger's scheme (1934) for $\beta \rightarrow \alpha$ applied in reverse, combined with the $\beta$ - $\omega$ plane collapse model (De Fontaine 1970). The $\beta$-lattice can be produced from the $\alpha$ unit cell by (i) $\sim 3 \%$ contraction along $[11 \overline{2} 0]_{a}$, (ii) $\sim 5 \%$ expansion along [1100], (iii) shift of the atom in the central plane along $[1010]_{a}$ by one sixth of its length and (iv) shear to decrease the hexagonal $120^{\circ}$ angle to $109^{\circ} 28^{\circ}$, the angle between $[111]_{\beta}$ and $[1 \overline{1} 1]_{\beta}$. The generation of the $(11 \overline{20})_{\omega}$ planes from the $(011)_{\beta}$ planes involves contractions of $\sim 1.1 \%$ along $[2 \overline{1} 1]_{\beta}$ direction (parallel to $[1100]_{a}$ ) and $\sim 1.8 \%$ along $[111]_{\beta}$ (parallel to $[1120]_{a}$ ) and movement of atoms marked $e$ and $f$ to $e^{\prime}$ and $f^{\prime}$ by $a_{\beta} \times \sqrt{3} / 12$, which is $\sim 0.48 \AA$ for $\mathrm{Ti}$.

\footnotetext{
*Another interesting observation is that the diffuse streaking decreases after pressure. This is against some recent theoretical models (for more details, see Sikka et al 1981).
} 


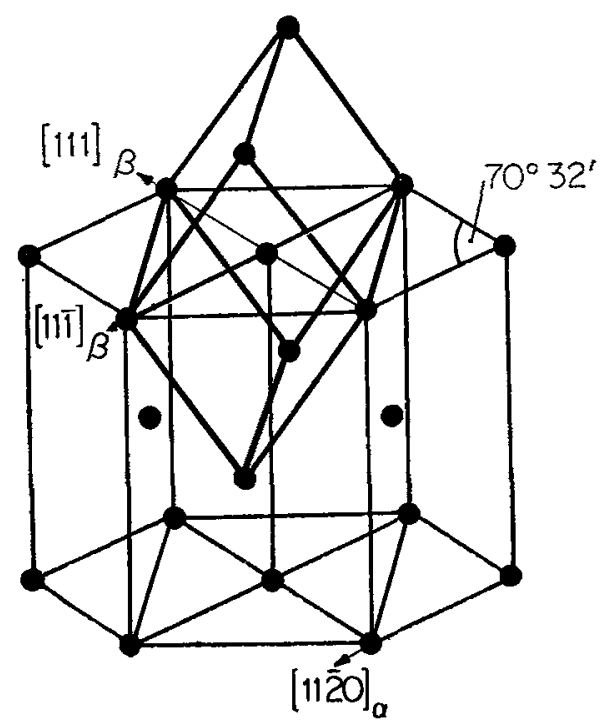

(o)

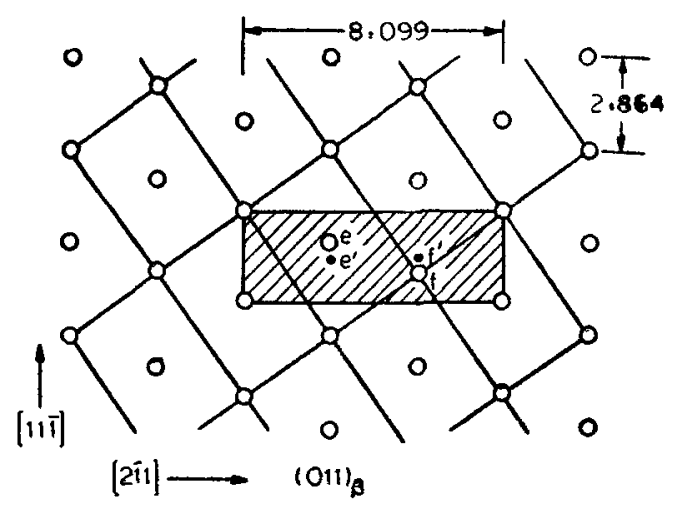

(b)

Figure 4. Relation of (a) $a$ and $\beta$ lattices and (b) $\beta$ and $\omega$ lattices.

In conclusion, our results show that the postulation of $\beta$ state as an intervening step during $\alpha \rightarrow \omega$ transition is correct. It is also consistent with the observations of Ageyev et al (1973) that in the decomposition of $(\beta+\omega)$-Ti alloys on ageing, the precipation of the $\alpha$-phase is preceded by partial dissolution of the $\omega$-phasein $\beta$ and there is no direct $\omega \rightarrow \alpha$ transition. The orientation relations observed by Flower and Swann (1973) which they take as evidence for Silcock's $\omega \rightarrow \alpha$ mechanism, also are consistent with the $\beta$-intermediate state.

\section{Acknowledgement}

Authors are grateful to Shri E S K Menon for collaboration in TEM studies. 


\section{References}

Afonikova N S, Degtyareva Y F, Litvin Yu A, Rabinkin A G and Skakov Yu A 1973 Sov. Phys. Solid State 15746

Ageyev N N, Petrova L V and Grankova L P 1973 in Titanium science and technology (eds) R I Jaffee and H M Burte (New York: Plenum) Vol. 3, p. 1495

Burgers W G 1934 Physica 1561

Collings E W 1975 J. Less-Common Met. 3963

Defontaine D 1970 Acta Metal 18275

Flower H M and Swann P R 1973 in Titanium science and technology (eds) R I Jaffee and H M Burte (New York: Plenum) Vol. 3, p. 1507

Hickman B S 1969 J. Mater. Sci. 4554

Jamieson J C 1963 Science 14072

Moss S C, Keating D T and Axe J D 1973 Solid State Commun. 131465

Sikka S K, Vohra Y K and Chidambaram R 1980 Solid State Commun. (in Press)

Silcock J M 1958 Acta Metal 6481

Usikov M P and Zilbershtein V A 1973 Phys. Status. Solidi $A 1953$

Vohra Y K 1978 J. Nucl. Mater. 75288

Vohra Y K, Menon E S K, Sikka S K and Krishnan R 1980 Acta Metal 29457

Williams J C 1973 in Titanium science and technology (eds) R I Jaffee and H M Burte (New York:

Plenum) Vol 3 p. 1433 